systems of surveillance. The distinction between such systems and surveys is not always appreciated.

As a rule, surveillance cannot hope to provide true population based estimates of injury occurrence. This is so because surveillance systems exist to monitor events and thus reveal new trends or unexpected occurrences. Rarely are these systems based on samples of events that are representative of what is happening in the population in which they are situated. For example, the CHIRPP system described by Stone and Doraiswamy in this issue (p 47), both in Glasgow and across Canada, is hospital based and not all injured children are treated in hospital. Even if it is reasoned that it is only injuries of sufficient severity to require hospital attention that are of interest, those treated in hospitals that are not part of the system will be missed. Thus, those 'captured' by the system are likely to reflect a somewhat biased sample even of all hospital treated injuries. Even in cases such as the NEISS system described by Weiss in this issue, or the UK's admirable home accident (HASS) or leisure accident (LASS) systems, where a studious attempt is made to include a representative sample of hospitals, such problems will inevitably remain.

Accordingly, surveillance results are best used to monitor trends or to make comparisons of injuries within the system itself. Under some circumstances the data may, however, also be used to evaluate program initiatives. Any such analyses are potentially of great value and accordingly, attempts to establish even modest, community based surveillance procedures will, almost inevitably, be rewarded. Moreover, they need not be costly. However, as the experience in Glasgow indicates, much groundwork needs to be laid to ensure cooperation and adequate support. Part of the secret is not to attempt to capture more information than is essential, and, where possible, to make the system fit well into the hospital's ordinary operating procedures - for example, by making it a part of administrative or accounting operations.

\title{
The Second BC Injury Prevention Conference
}

The Second BC Injury Prevention Conference in Vancouver, BC held Nov 23-25 was inspirational. A dispassionate account of it may make it appear little different than most such conferences. But in this case, perhaps because I went expecting 'more of the same', the experience was surprisingly rewarding. I use this opportunity to convey some of the passion I felt at various moments during this unusual event.

First, I was impressed that it was the second such conference. Too often similar gatherings are one-off affairs; the organizers don't see the need to do it again, or don't have the energy to do so. But by bringing much the same group of persons together at regular intervals, not necessarily annually, a sense of corporate spirit is built. In part this is simply a result of establishing personal contacts with other, kindred spirits.

Second, I was not prepared for the exceptionally diverse range of participants and sponsors. This alone speaks volumes about the extent to which the injury prevention message has infiltrated so many levels of society, including the corporate and government sectors. For example, the co-chair was a fire department official who is responsible for an excellent, mobile, nearly full scale home safety display.

Finally, there were a number of highlights. Apart from a typically solid, straightforward, and sensible presentation by Fred Rivara, and a humorous lunch talk by a fire inspector with a serious message, there was a brief presentation by a representative of the Ministry of Transport. In this she announced a number of no-holds-barred tough road safety measures that fully convinced me that this province is genuinely determined to reduce traffic injuries to pedestrian and cyclists. (It is not, therefore, incidental that $\mathrm{BC}$ is the first province to have a mandatory bicycle helmet law with teeth.)

Perhaps this talk should not have been such a surprise because this province has a brief but impressive record of taking injury prevention seriously. For several years BC has had an Office for Injury Prevention (OIP) in the Ministry of Health. This reflects, at least in part, the point I struggled to make in an earlier editorial about injury prevention being a health responsibility. At least this constituency appears to agree.

Continuing on my note of pleasant surprise, the second edition of a directory produced by the OIP lists more than 140 organizations and agencies involved in injury prevention. They cover the alphabet: from the Aboriginal Health Association of BC, BC Automobile Association and BC Children's Hospital's, Safe Start injury prevention program to McDonald's Restaurants, the National Bicycle Safety Foundation, police and fire departments, the Vancouver Health Department and the Whistler Sports Injury Prevention Program. Many of these were represented at the conference itself.

I admit I have had occasion to point to lists such as this and say that with this many players the need for coordination is immense and that someone or some group must take the lead. British Columbia's Minister of Health, the Honourable Paul Ramsey, has done just that, appointing the Minister's Injury Prevention Advisory Committee with the mandate to develop a coordinated comprehensive approach in order to reduce unintentional injuries in British Columbia. The OIP as secretariat plays a supporting role.

All right. It rained for three days and there were some disappointing moments. Far and away the greatest disappointment was my realization that apart from a handful of the speakers there were few, if any, attendees who were health professionals. I will refrain from editorializing further about what I think this means save to say that many health professionals still fail to understand the nature and seriousness of the injury epidemic. 